\title{
Angiostrongylus cantonensis in North African hedgehogs as vertebrate hosts, Mallorca, Spain, October 2018
}

Claudia Paredes-Esquivel ${ }^{1}$, Jessica Sola ${ }^{2}$, Sofía Delgado-Serra ${ }^{1}$, Miguel Puig Riera², Nieves Negre², Miguel Ángel Miranda ${ }^{1}$, José A Jurado-Rivera ${ }^{3}$

1. Applied Zoology and Animal Conservation Group, University of the Balearic Islands, Palma de Mallorca, Spain

2. Centre de Recuperació de Fauna Silvestre de les Illes Balears (COFIB), Mallorca, Spain

3. Laboratory of Genetics, University of the Balearic Islands, Palma de Mallorca, Spain

Correspondence: Claudia Paredes-Esquivel (claudia.paredes@uib.es)

Citation style for this article:

Paredes-Esquivel Claudia, Sola Jessica, Delgado-Serra Sofía, Puig Riera Miguel , Negre Nieves, Miranda Miguel Ángel, Jurado-Rivera José A. Angiostrongylus cantonensis in North African hedgehogs as vertebrate hosts, Mallorca, Spain, October 2018. Euro Surveill. 2019;24(33):pii=1900489. https://doi. org/10.2807/1560-7917.ES.2019.24.33.1900489

In October 2018, two Atelerix algirus hedgehogs were admitted to the Wildlife Rehabilitation Hospital in Mallorca (Balearic Islands, Spain) with signs of acute neurological disease. Necropsy detected immature, fully developed nematodes in the subarachnoid space of both hedgehogs, including a gravid female worm. DNA-based molecular tools confirmed the nematode as Angiostrongylus cantonensis, an important aetiological agent of eosinophilic meningitis in humans. So far this zoonotic parasite in has not been reported in western European wildlife.

We report the presence of the rat lungworm, Angiostrongylus cantonensis, in two Atelerix algirus (North African) hedgehogs taken from two different localities on Majorca (Balearic Islands, Spain). This report highlights the importance of using wildlife hosts as biosentinels of potentially emerging zoonosis.

\section{Clinical and pathological findings in the hedgehogs}

In October 2018, two adult (one male and one female) $A$. algirus hedgehogs were admitted to the Wildlife Rehabilitation Centre (COFIB) in Mallorca (Balearic Islands, Spain) from two different locations on the island. The animals showed signs of an acute central nervous system disorder. Symptoms included pelvic limb ataxia, atonia, posterior paresis of thoracic limbs and behavioural decay. Clinical manifestations progressed over a few days and hedgehogs were euthanised (female 1 day and male 10 days after admittance) to avoid any further suffering. Necropsy revealed the presence of nematode worms in the subarachnoid space of the brain in both hedgehogs. The female hedgehog was infected with a single nematode larva
(11.1 $\mathrm{mm}$ long) and presented no perceptible lesions of the pia mater. The male hedgehog was infected with five fully developed nematodes, including a gravid female and one immature nematode; the pia mater exhibited lesions compatible with multifocal haemorrhage (Figure 1).

Adult specimens extracted from the male hedgehog measured $15^{-17} \mathrm{~mm}$ in length. Morphological examination following the dichotomous keys by Kinsella [1] suggested the nematodes were $A$. cantonensis. Male nematodes showed a copulatory bursa (Figure $2 \mathrm{~A}$ ) and gravid females showed a 'barber's pole' appearance, blood-containing intestine and the typical morphology of the tail (Figure 2B).

\section{Molecular identification of nematode species}

The species identity of the nematodes was confirmed by PCR and sequencing of the Cytochrome Oxidase/ gene region from three specimens, using primers $\mathrm{COI}$ F: 5'- TTTTTTGGGCATCCTGAGGTTTAT -3' and COI R: 5'- TAAAGAAAGAACATAATGAAAATG 3' [2]. PCR reactions were prepared in a total volume of $25 \mu \mathrm{L}$ containing $17.2 \mu \mathrm{L}$ of water, $2.5 \mu \mathrm{L}$ of $10 \times$ Bioline PCR buffer, $1.75 \mu \mathrm{L}$ of $\mathrm{MgCl}_{2}$ (50mM), $1 \mu \mathrm{L}$ of dNTP mix (10mM total), $0.5 \mu \mathrm{L}$ of each primer $(100 \mu \mathrm{M}), 0.5 \mu \mathrm{L}$ of BSA $(20 \mathrm{mg} / \mathrm{mL}$; New England BioLabs, Hitchin, Hertfordshire, United Kingdom), 0.05 $\mu \mathrm{L}$ of Bioline Taq DNA polymerase and $1 \mu \mathrm{L}$ of template DNA. Reactions were incubated at $94^{\circ} \mathrm{C}$ for 5 mins, followed by 35 cycles at $94^{\circ} \mathrm{C}$ for $30 \mathrm{~s}$, $48^{\circ} \mathrm{C}$ for $30 \mathrm{~s}$ and $72^{\circ} \mathrm{C}$ for $45 \mathrm{~S}$ and a final extension step at $72{ }^{\circ} \mathrm{C}$ for 10 mins. PCR products were visualised on $1.5 \%$ agarose gel electrophoresis stained with ethidium bromide and purified using MSB Spin PCRapace 


\section{FIGURE 1}

Ventral view of the brain of a male Atelerix algirus hedgehog, Mallorca, Spain, 2018

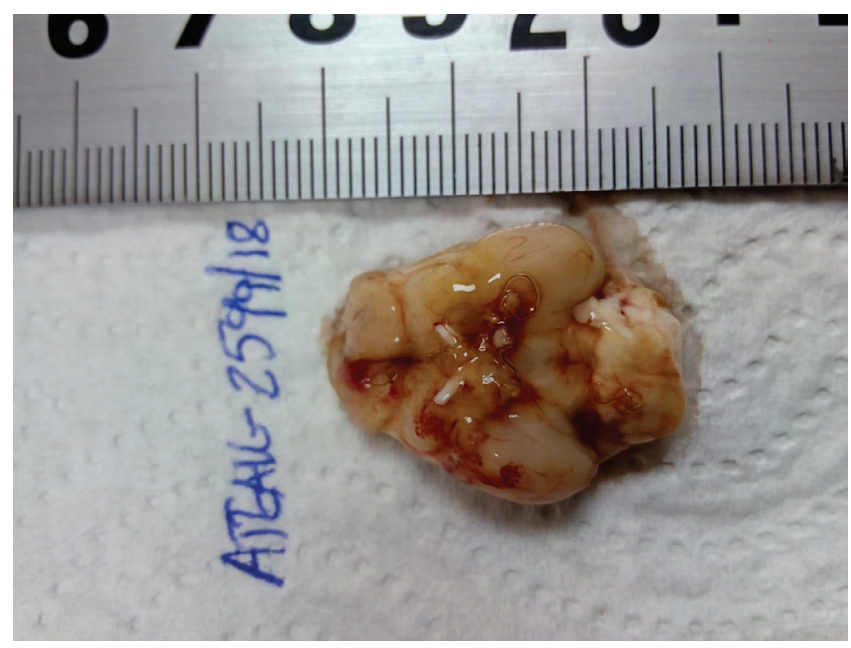

Several nematode specimens and lesions compatible with multifocal haemorrhage can be observed.

(Invitek, Berlin, Germany) for subsequent sequencing using the BigDye Terminator Cycle Sequencing kit (Applied Biosystems, Foster City, California, United States). Three PCR amplifications were conducted on individual specimens. All three sequenced samples resulted in the same COI haplotype. This DNA sequence was blasted against the GenBank database and the top 57 hits corresponded with $\mathrm{CO}$ / sequences of $A$. cantonensis, with the first four alignments yielding a $100 \%$ identity match with specimens from Australia, Tenerife, Taiwan and New Orleans (US), respectively (Figure 3). The sequence has been submitted to the GenBank website (accession number MN227185) and two adult nematode specimens and the DNA extractions used in this study have been kept as vouchers at $-80^{\circ} \mathrm{C}$ in the laboratory of zoology of the University of the Balearic Islands for further investigation.

\section{Discussion}

Angiostrongyliasis is an emerging zoonosis [3] caused by the metastrongyloid nematode Angiostrongylus cantonensis. Rats are the definitive hosts of this parasitic nematode, while birds and mammals act as accidental hosts. Infection typically occurs after vertebrates ingest intermediate hosts (snails and slugs) or paratenic hosts (freshwater shrimps, crabs, flatworms and frogs). Gastropod mucus may also be involved in the transmission cycle [4]. Severe clinical manifestations have been reported in vertebrate hosts, due to the parasite's tropism to the central nervous system [5]. In humans, it is a leading cause of parasitic eosinophilic meningitis [6]. Angiostrongyliasis eosinophilic meningitis (AEM) is difficult to diagnose [3] and requires a high degree of clinical suspicion since eosinophilia may not be initially observed in peripheral blood [7] and serology may be misleading [8]. In areas where angiostrongyliasis is not endemic, AEM may thus be overlooked [9].

Human angiostrongyliasis is endemic to the Caribbean, Pacific Islands and Asia and has been shown to spread to temperate and sub-tropical regions [9], with about 3,000 cases reported in 30 countries [3]. Infected rats carried by ships have been suggested as the main agents of parasite expansion in the Asia-Pacific region after World War II [10] and may still be implicated in its further spread. Since the detection of angiostrongyliasis in rats in the Atlantic island of Tenerife (Canary Island, Spain) further spread to Europe has been anticipated by some [11]. However, to date, only a single autochthonous human case of angiostrongyliasis has been reported from France in 2016. Imported food could not be ruled out as a possible source of contamination, local active transmission could not be confirmed [12].

Hedgehogs are zoonotic agents of various parasitic diseases [13], but they have never been shown to act as reservoir hosts for parasites of the genus Angiostrongylus [5]. We had previously observed hedgehogs with clinical manifestations compatible with acute neurological disease, but necropsy did not warrant examination of parasitic nematodes in the brain, as no previous reports of nematodes had been previously reported in hedgehogs. The North African hedgehog, is widely distributed in the western Mediterranean Basin and in the Canary Islands. It is the most common mammal species hospitalised at the COFIB and its diet mainly consists of insects and snails. Most hedgehog fatalities at the centre are due to another gastropodborne lungworm, Crenosoma striatum [14].

The presence of an adult gravid female nematode in the hedgehog's brain was an interesting finding, as in accidental hosts only the sub-adult (fifth) stage has been found in the central nervous system [15]; moreover, our specimens were slightly smaller than those found in the pulmonary arteries of rats (size range 18.5-33) [16]. We did not search for adult worms in the pulmonary arteries of hedgehogs and we did not conduct any survey in rats in the island. These are the main limitations of this study. Further investigations should clarify the role of hedgehogs in the life cycle of $A$. cantonensis.

The Balearic hedgehog populations are geographically isolated and two specimens from different locations were infected suggesting that active transmission of $A$. cantonensis is occurring on the island; this is the first report of $A$. cantonensis infecting western European wildlife. Majorca has strong connection by sea and air with mainland Europe and millions of tourists visit the island each year (e.g. 16.5 million in 2018) [17], this and the ubiquity of rats and snails on the island could facilitate a spread of $A$. cantonensis.

Snails are an important part of the Majorcan cuisine and they are commercialised in heliciculture farms on 


\section{FIGURE 2}

Angiostrongylus cantonensis views of (A) copulatory bursa of male specimens supported by bursal rays and (B) a female tail end, Spain, 2018

A. Copulatory bursa, male specimens with bursal rays

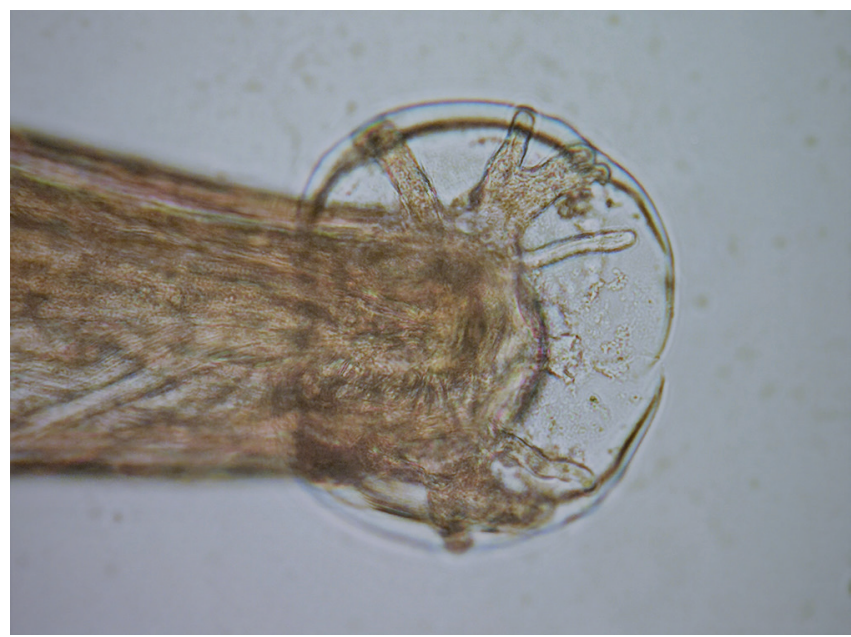

B. Female tail end

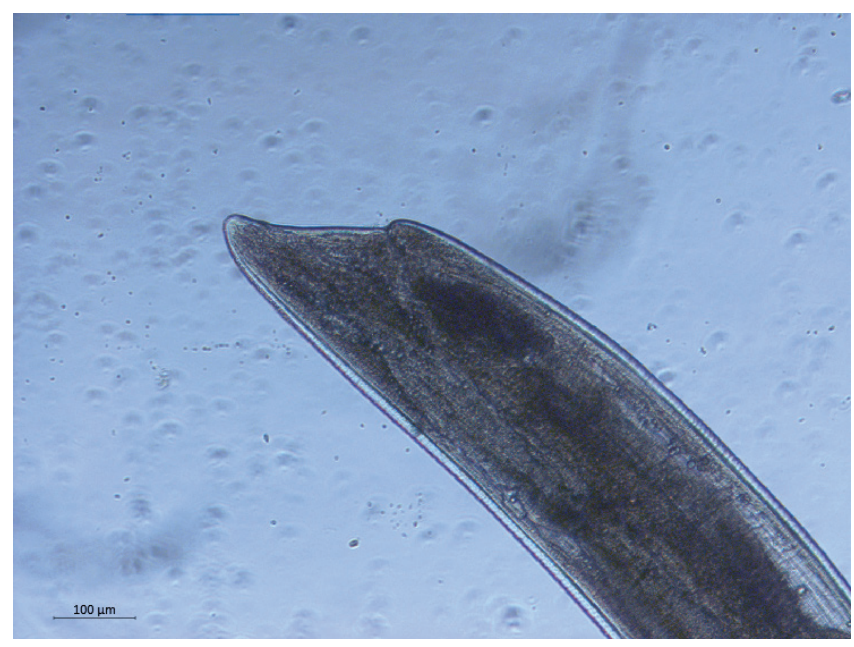

Spiculae are not evident since voucher specimens have not been treated with clearing solutions.

the island or inhabitants can collect wild snails from open fields. It is therefore, beneficial to determine which intermediate host species are involved in the transmission of $A$. cantonensis. A previous report in Tenerife indicated that the garden snail (Cornu aspersum) and Mediterranean snail (Theba pisana) are competent vectors of $A$. cantonensis [18]; these species are also present in Balearic Islands. The best way to prevent human infections is by not eating raw or undercooked snails or freshwater shrimps or other paratenic hosts. However, pieces of snails accidently eaten in inadequately washed/cooked vegetables have been associated with possible cases of eosinophilic meningitis [4]. The risk of human infection could be avoided if control measures e.g. wearing gloves when handling snails/slugs, washing hands are undertaken [19]. Additional measures that could be taken are increasing public awareness of $A$. cantonensis and surveillance of vectors and vertebrate hosts in endemic areas.

\section{Conclusions}

This report highlights the importance of using wildlife hosts as biosentinels for possible emerging zoonotic infections and the need to consider $A$. cantonensis as potential aetiological agent of eosinophilic meningoencephalitis within western Europe. 


\section{FIGURE 3}

Neighbour-joining tree based on the sequence from the Cytochrome Oxidase I gene region from three nematode specimens, Spain, 2018

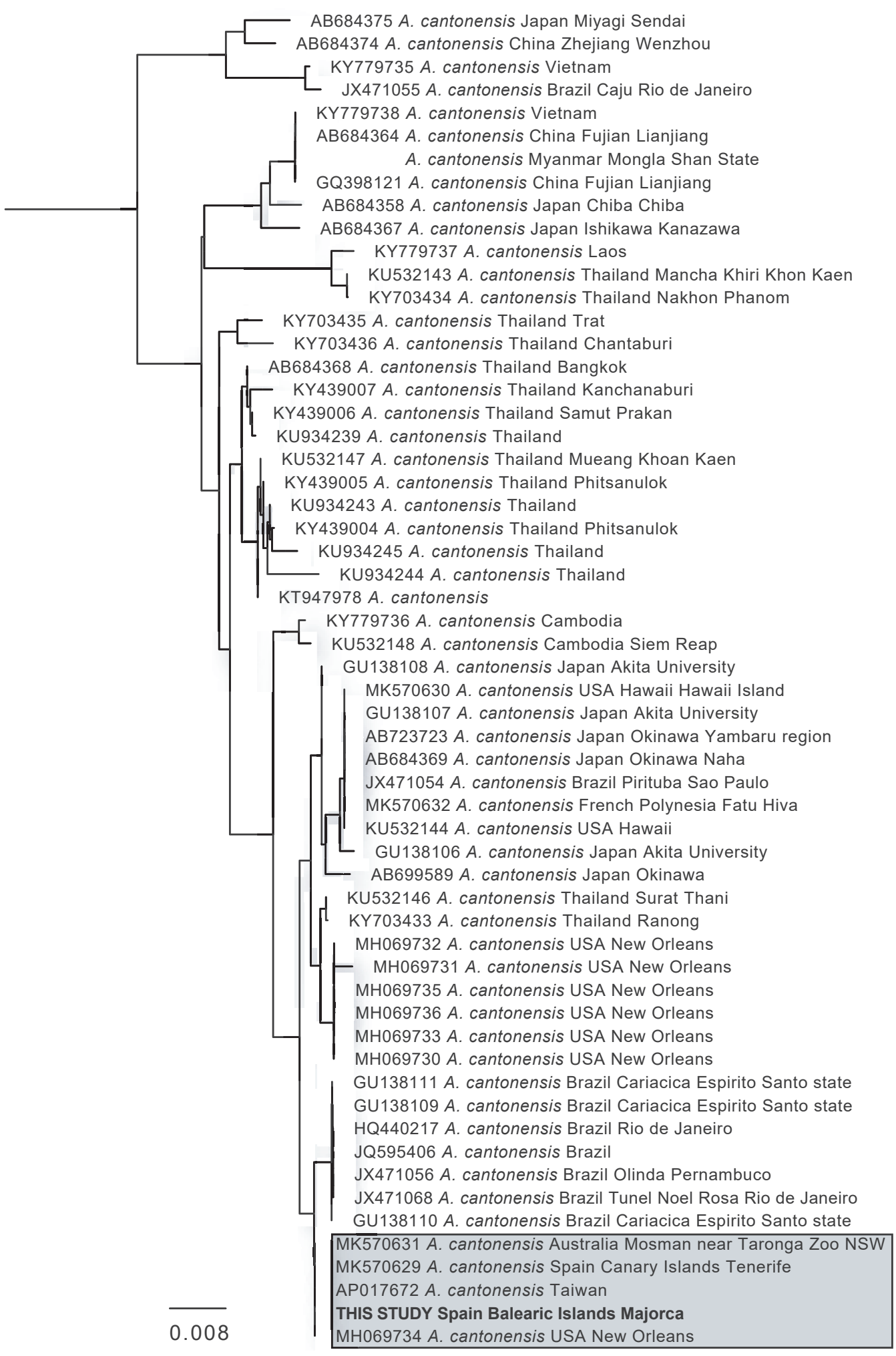

The most similar sequences retrieved from GenBank after using the haplotype from the Balearic Islands in a BLAST search were used to generate a Neighbour-joining tree by selecting the 'distance tree of results' option implemented in the BLAST server. The geographical source of the sequences has been appended to the taxa names when available. 


\section{Acknowledgements}

The authors would like to thank Dr. Deidre Walshe, Dr. Lee Haines, Dr. Luis Laglera, Dr. Ursula Paredes and Dr. Luis Parpal, for their thoughtful criticisms and comments to this manuscript.

\section{Conflict of interest}

None declared.

\section{Authors' contributions}

CPE designed the study, identified specimens morphologically, analysed results and wrote first draft. JS, NN and MPR conducted field work, clinical examinations and necropsies. SDS identified specimens morphologically, MAM was involved in the analysis of the data, JAJ contributed to the design of the study and performed molecular and phylogenetic analysis. All authors contributed to the edition of the manuscript.

\section{References}

1. Kinsella JM. Angiostrongylus schmidti sp. n. (Nematoda: Metastrongyloidea) from the rice rat, Oryzomys palustris, in Florida, with a key to the species of Angiostrongylus Kamensky, 1905. J Parasitol. 1971;57(3):494-7. https://doi. org/10.2307/3277901 PMID: 5104560

2. Monte TCC, Simões RO, Oliveira APM, Novaes CF, Thiengo SC, Silva AJ, et al. Phylogenetic relationship of the Brazilian isolates of the rat lungworm Angiostrongylus cantonensis (Nematoda: Metastrongylidae) employing mitochondrial COI gene sequence data. Parasit Vectors. 2012;5(1):248. https:// doi.org/10.1186/1756-3305-5-248 PMID: 23130987

3. Cowie RH. Angiostrongylus cantonensis: agent of a sometimes fatal globally emerging infectious disease (rat lungworm disease). ACS Chem Neurosci. 2017;8(10):2102-4. https://doi. org/10.1021/acschemneuro.7bo0335 PMID: 28902487

4. Cowie RH. Pathways for transmission of angiostrongyliasis and the risk of disease associated with them. Hawaii J Med Public Health. 2013;72(6) Suppl 2;70-4. PMID: 23901388

5. Spratt DM. Species of Angiostrongylus (Nematoda: Metastrongyloidea) in wildlife: A review. Int J Parasito Parasites Wildl. 2015;4(2):178-89. https://doi.org/10.1016/j. ijppaw.2015.02.006 PMID: 25853051

6. Graeff-Teixeira C, da Silva ACA, Yoshimura K. Update on eosinophilic meningoencephalitis and its clinical relevance. Clin Microbiol Rev. 2009;22(2):322-48. https://doi. org/10.1128/CMR.00044-08 PMID: 19366917

7. Slom TJ, Cortese MM, Gerber SI, Jones RC, Holtz TH, Lopez AS, et al. An outbreak of eosinophilic meningitis caused by Angiostrongylus cantonensis in travelers returning from the Caribbean. N Engl J Med. 2002;346(9):668-75. https://doi. org/10.1056/NEJMoa012462 PMID: 11870244

8. Brummaier T, Bertschy S, Arn K, Treumann T, Ruf M-T, Nickel $B$, et al. A blind passenger: a rare case of documented seroconversion in an Angiostrongylus cantonensis induced eosinophilic meningitis in a traveler visiting friends and relatives. Trop Dis Travel Med Vaccines. 2019;5(1):6. https:// doi.org/10.1186/S40794-019-0084-X PMID: 31016026

9. Barratt J, Chan D, Sandaradura I, Malik R, Spielman D, Lee R, et al. Angiostrongylus cantonensis: a review of its distribution, molecular biology and clinical significance as a human pathogen. Parasitology. 2016;143(9):1087-118. https://doi. org/10.1017/So031182016000652 PMID: 27225800

10. Kliks MM, Palumbo NE. Eosinophilic meningitis beyond the Pacific Basin: the global dispersal of a peridomestic zoonosis caused by Angiostrongylus cantonensis, the nematode lungworm of rats. Soc Sci Med. 1992;34(2):199-212. https:// doi.org/10.1016/0277-9536(92)90097-A PMID: 1738873

11. Foronda P, López-González M, Miquel J, Torres J, Segovia $\mathrm{M}$, Abreu-Acosta N, et al. Finding of Parastrongylus cantonensis (Chen, 1935) in Rattus rattus in Tenerife, Canary Islands (Spain). Acta Trop. 2010;114(2):123-7. https://doi. org/10.1016/j.actatropica.2010.02.004 PMID: 20153283

12. Nguyen Y, Rossi B, Argy N, Baker C, Nickel B, Marti H, et al. Autochthonous case of eosinophilic meningitis caused by Angiostrongylus cantonensis, France, 2016. Emerg Infect Dis.
2017;23(6):1045-6. https://doi.org/10.3201/eid2306.161999 PMID: 28518042

13. Riley PY, Chomel BB. Hedgehog zoonoses. Emerg Infect Dis. 2005;11(1):1-5. https://doi.org/10.3201/eid1101.040752 PMID: 15705314

14. Garcia-Salguero A, Delgado-Serra S, Sola J, Negre N, Miranda MA, Paredes-Esquivel C. Combined morphology and DNAbarcoding to identify Plagiorhynchus cylindraceus cystacanths in Atelerix algirus. Parasitol Res. 2019;118(5):1473-8. https:// doi.org/10.1007/s00436-019-06299-6 PMID: 30927061

15. Cowie RH. Biology, systematics, life cycle, and distribution of Angiostrongylus cantonensis, the cause of rat lungworm disease. Hawaii J Med Public Health. 2013;72(6) Suppl 2;6-9. PMID: 23901372

16. Mackerras MJ, Sandars DF. MJ M. DF S. The life history of the rat lung-worm, Angiostrongylus cantonensis (Chen) (Nematoda: Metastrongylidae). Aust J Zool. 1955;3(1):1-21. https://doi.org/10.1071/Z09550001

17. AETIB. El turisme a les Illes Balears. Anuari 2018. Palma de Mallorca: Agència d'Estratègia Turística de les Illes Balears; 2018.

18. Martin-Alonso A, Abreu-Yanes E, Feliu C, Mas-Coma S, Bargues MD, Valladares B, et al. Intermediate hosts of Angiostrongylus cantonensis in Tenerife, Spain. PLoS One. 2015;10(3):e0120686. https://doi.org/10.1371/journal. pone.0120686 PMID: 25803658

19. Centres for Disease Control and Prevention (CDC). Parasites - Angiostrongyliasis (also known as Angiostrongylus Infection). Epidemiology \& Risk Factors. Angiostrongylus cantonensis. Atlanta: CDC; 2018. Available from: https://www.cdc.gov/parasites/angiostrongylus/epi. html\#targetText=Outbreaks\%20of\%2ohuman $\% 20$ angiostrongyliasis $\% 2$ have, literature $\% 2$ from $\% 20$ approximately $\% 2030 \% 20$ countries

\section{License, supplementary material and copyright}

This is an open-access article distributed under the terms of the Creative Commons Attribution (CC BY 4.0) Licence. You may share and adapt the material, but must give appropriate credit to the source, provide a link to the licence and indicate if changes were made.

Any supplementary material referenced in the article can be found in the online version.

This article is copyright of the authors or their affiliated institutions, 2019. 